TOPOLOGICAL ALGEBRAS, THEIR APPLICATIONS,

AND RELATED TOPICS

BANACH CENTER PUBLICATIONS, VOLUME 67

INSTITUTE OF MATHEMATICS

POLISH ACADEMY OF SCIENCES

WARSZAWA 2005

\title{
SPECTRAL ISOMETRIES
}

\author{
MARTIN MATHIEU \\ Department of Pure Mathematics, Queen's University Belfast \\ Belfast BT7 1NN, Northern Ireland \\ E-mail:m.m@qub.ac.uk
}

Dedicated to Professor Wiesław Żelazko on his 70th birthday

\begin{abstract}
In this survey, we summarise some of the recent progress on the structure of spectral isometries between $C^{*}$-algebras.
\end{abstract}

A fundamental question in physics asks to what extent the algebraic model one chooses for a given physical system is predetermined by the geometric data obtained from the system. In a quantum mechanical system the data are the measurements, that is, the spectral values of the observables, which are represented by self-adjoint operators on some Hilbert space. Following the approach by Jordan, von Neumann and Wigner [7] the mathematical model is the one of a Jordan algebra. Therefore the question reads in this case: If all spectral values of the observables in a quantum mechanical system are known, will the algebraic model be uniquely determined up to Jordan isomorphism?

In [9], Kaplansky phrased this question mathematically rigorously in the following way: Suppose that $T: A \rightarrow B$ is a linear surjection between semisimple unital complex Banach algebras $A$ and $B$. Suppose that $T 1=1$ and that, for every $x \in A$, Tx is invertible if and only if $x$ is invertible. Is T necessarily a Jordan isomorphism? For a nice exposition on the history and the plentiful results on Kaplansky's question, we refer the reader to Aupetit's survey in [3]. Among the many contributions is the Gleason-KahaneŻelazko theorem stating that every unital invertibility-preserving linear mapping into a commutative semisimple Banach algebra must be multiplicative; see [20].

For an element $x$ in a unital Banach algebra $A$, let $\sigma(x)$ denote its spectrum. We call a linear mapping $T: A \rightarrow B$ spectrum-preserving if $\sigma(T x)=\sigma(x)$ for all $x \in A$. It is easy to verify that, for surjections between semisimple algebras, this assumption is equivalent to

2000 Mathematics Subject Classification: Primary 47A65; Secondary 46L10, 47A10, 47B48.

Key words and phrases: spectral isometry, Jordan isomorphism, von Neumann algebra.

This paper is part of the research carried out in the EC network Analysis and Operators (HPRN-CT-2000-00116).

The paper is in final form and no version of it will be published elsewhere. 
$T 1=1$ ( $T$ is unital) and $T x$ invertible if and only if $x$ invertible. Therefore the following result answers Kaplansky's question positively in the context of von Neumann algebras.

THEOREM 1 [4]. Let $T$ be a surjective spectrum-preserving linear mapping between the von Neumann algebras $A$ and $B$. Then $T$ is a Jordan isomorphism.

Since von Neumann algebras are, to a large extent, the algebras of operators occurring in the algebraic formalism of quantum mechanics, Aupetit's result provides indeed an elegant and satisfactory answer to the original question. Despite this fact, it has its drawbacks. The main one probably is that one needs to know all measurements of all observables. This is in general a difficult task. It is therefore close at hand to try to weaken the assumption to preservation of only part of the spectrum or possibly only one spectral value. We shall here take the view that the spectral radius (i.e., in a sense the "largest eigenvalue") should be sufficient to give the same result. In this article, we will report on some recent progress made in this direction.

For an element $x$ in a Banach algebra, let

$$
r(x)=\sup \{|\lambda| \mid \lambda \in \sigma(x)\}=\lim _{n \rightarrow \infty}\left\|x^{n}\right\|^{1 / n}
$$

denote the spectral radius of $x$. We say that $T: A \rightarrow B$ is spectrally bounded if it is linear and there exists a constant $M \geq 0$ such that, for all $x \in A, r(T x) \leq M r(x)$. We say that $T$ is a spectral isometry if $r(T x)=r(x)$ for all $x \in A$.

Every bounded linear operator from a commutative $C^{*}$-algebra is spectrally bounded; thus it is impossible to hope for a description of spectrally bounded operators on arbitrary $C^{*}$-algebras. The situation, however, changes once we impose suitable infiniteness conditions on the domain; see [11] and [13]. In the following, we shall focus our attention on spectral isometries. Several of their basic properties are compiled in [12, Section 4]; we shall use them in the sequel without further reference.

Suppose that $T: A \rightarrow B$ is a spectral isometry from the $C^{*}$-algebra $A$ into the commutative $C^{*}$-algebra $B$. By [10, Lemma 2.1], $T(x y)=T(y x)$ for all $x, y \in A$ and, since $T$ is injective, we conclude that $A$ is commutative as well. The Banach-Stone theorem thus yields the following version of the Gleason-Kahane-Żelazko theorem for spectral isometries.

Proposition 2. Every unital spectral isometry from a unital $C^{*}$-algebra onto a commutative unital $C^{*}$-algebra is multiplicative.

Let us now change the assumption on the codomain. Suppose that $B$ is finite dimensional. Then $A$ must be finite dimensional as well; hence both are finite direct sums of full matrix algebras. Supposing that $T$ is onto, we derive the following description.

Proposition 3. Every unital spectral isometry from a unital $C^{*}$-algebra onto a finite dimensional $C^{*}$-algebra is a Jordan isomorphism.

This is a consequence of [14, Corollary 5], which in turn rests on the earlier result for $A=B=M_{n}(\mathbf{C})$ obtained in [2, Proposition 2] and the following fundamental compatibility of spectral isometries with central decompositions. 
Proposition 4 [14, Proposition 2]. Let $T: A \rightarrow B$ be a unital surjective spectral isometry, $e \in A$ a central projection, and put $f=T e$. Then $f$ is a central projection in $B$ and the restriction $T_{e}$ of $T$ to $e A$ is a unital spectral isometry onto $f B$.

These observations together with Theorem 1 made us surmise the following in [12].

CONJECTURE. Every unital surjective spectral isometry between unital $C^{*}$-algebras is a Jordan isomorphism.

Note that a positive answer to this conjecture would give a far-reaching generalisation of Aupetit's theorem. It is well known that a Jordan isomorphism is unital and invertibility-preserving; hence it preserves spectra and a fortiori is a spectral isometry. We are therefore seeking a spectral radius characterisation of Jordan isomorphisms. This can also be seen as a non-selfadjoint analogue of Kadison's famous non-commutative extension of the Banach-Stone theorem: every unital surjective isometry between unital $C^{*}$-algebras is a Jordan *-isomorphism.

However, at this moment, the tools available do not seem to take us much beyond the setting of von Neumann algebras. In this framework, we have the following result.

THEOREM 5. Every unital spectral isometry from a von Neumann algebra onto a von Neumann algebra without direct summand of type $\mathrm{II}_{1}$ is a Jordan isomorphism.

By symmetry, the hypothesis on the codomain could be put on the domain instead. The remainder of this article will be devoted to describing the essential ideas that go into the proof of Theorem 5 .

Proof of Theorem 5. Let $T: A \rightarrow B$ be such a spectral isometry. We shall use the decomposition of von Neumann algebras into various types according to Murray and von Neumann and refer the reader to [22] for details. We begin with decomposing $A$ into its finite and properly infinite part, $A=A_{\text {fin }} \oplus A_{\infty}$. Let $z$ be the maximal finite central projection in $A$ so that $A_{\text {fin }}=z A$. Since $T$ restricts to a ${ }^{*}$-isomorphism between the centres of $A$ and $B$ ([12, Corollary 4.4]), $z^{\prime}=T z$ is the maximal finite central projection in $B$ and, by Proposition 4 above, $T_{z}: A_{\text {fin }} \rightarrow z^{\prime} B=B_{\text {fin }}$ is a unital surjective spectral isometry. Likewise, $T_{1-z}$ is a unital spectral isometry from the properly infinite von Neumann algebra $A_{\infty}$ onto $B_{\infty}$. Clearly, $T$ is a Jordan homomorphism if and only if both $T_{z}$ and $T_{1-z}$ are Jordan homomorphisms.

The main result in [13] states that every unital surjective spectrally bounded operator $S$ from a properly infinite von Neumann algebra is a Jordan homomorphism. Its proof uses the fundamental fact that every surjective spectrally bounded operator is bounded, as observed by Aupetit in [1, Theorem 5.5.2], and it is thus sufficient to show that $S e$ is idempotent for every projection $e$ in the domain ([13, Lemma 2.1]). The crucial property, however, is that $S x$ is nilpotent of order $n$ for every $x$ with $x^{n}=0$ ([13, Lemma 3.1]), which rests on a spectral-radius characterisation of nilpotent elements due to Ransford and White [18]. This enables us to apply the result by Pearcy and Topping [16] that every properly infinite von Neumann algebra is linearly generated by elements of square zero. For more details, see [13, Theorem 3.6]. As a result, $S=T_{1-z}$ is a Jordan homomorphism. 
In order to analyse $T_{z}$, we further decompose $A_{\text {fin }}$ into subalgebras of type I and type II: $A_{\text {fin }}=A_{1} \oplus A_{2}$, where $A_{1}$ is a finite type I von Neumann algebra and $A_{2}$ is of type $\mathrm{II}_{1}$. Once again, there is a central projection $z_{1} \in A$ such that $A_{1}=z_{1} A$ and $A_{2}=\left(z-z_{1}\right) A$. As $T_{z}$ respects this central decomposition, and $T_{z-z_{1}}$ maps $A_{2}$ onto a direct summand of $B$ of type $\mathrm{II}_{1}$, our hypothesis allows us to assume that $T_{z}$ acts on a finite type I algebra. The claim is thus accomplished by [14, Theorem 11], but we will add a few comments on its proof here.

Every finite type I von Neumann algebra is a direct product of $n$-homogeneous von Neumann algebras each of which is of the form $C\left(X, M_{n}(\mathbf{C})\right)$ for some hyperstonean space $X$. We can therefore focus on von Neumann algebras of the latter kind, i.e. assume that $A=C\left(X, M_{n}(\mathbf{C})\right)$. Since $Z(A)=C(X)$, every Glimm ideal $I$ of $A$ is of the form

$$
I=\left\{f \in C\left(X, M_{n}(\mathbf{C})\right) \mid f(t)=0 \text { for some } t \in X\right\} .
$$

One of the nice properties of $T$ is that is respects Glimm ideals, that is, $J=T I$ is a Glimm ideal in $B$ as well ([14, Proposition 10]). By means of this, we obtain an induced operator $\hat{T}: \hat{A}=A / I \rightarrow \hat{B}=B / J$. The spectral radius formula in quotient $C^{*}$-algebras (see [17, Theorem 2] or [15, Corollary on p. 274]) enables us to deduce that $\hat{T}$ is a unital surjective spectral isometry ([14, Proposition 9]). By the above, $\hat{A}$ is isomorphic to $M_{n}(\mathbf{C})$ so $\hat{B}$ is finite dimensional as well. Since $\hat{B}$ is primitive by Halpern's result ([6, Theorem 4.7]), it follows that $\hat{B}$ is also isomorphic to $M_{n}(\mathbf{C})$, so that we are back to the case of $n \times n$ matrices, which was already treated (compare Proposition 3). Recall that the Glimm ideals separate the points of $A$; this entails that $T$ is a Jordan isomorphism under the assumption that $A$ is a finite type I von Neumann algebra.

Consequently, $T_{z}$ is a Jordan isomorphism too, which completes the proof.

Remarks. 1. Some of the arguments in the proof of Theorem 5 extend to more general $C^{*}$-algebras. For instance, the application of the spectral theorem in the first paragraph also works in every $C^{*}$-algebra of real rank zero. Properly infinite von Neumann algebras can be replaced by purely infinite simple unital $C^{*}$-algebras, as observed in [11]. Thus the argument using Glimm ideals also works in the case $A=C(X, C)$, where $X$ is totally disconnected compact and $C$ a purely infinite simple unital $C^{*}$-algebra. However, this does not seem to lead to a strategy to tackle the situation of general $C^{*}$-algebras.

2. The case of infinite type I factors had been treated earlier by Brešar and Šemrl [5]; see also [19].

\section{References}

[1] B. Aupetit, A Primer on Spectral Theory, Springer-Verlag, New York, 1991.

[2] B. Aupetit, Spectral characterization of the radical in Banach and Jordan-Banach algebras, Math. Proc. Camb. Phil. Soc. 114 (1993), 31-35.

[3] B. Aupetit, Sur les transformations qui conservent le spectre, in: Banach Algebras 1997, E. Albrecht and M. Mathieu (eds.), Walter de Gruyter, Berlin, 1998, 55-78.

[4] B. Aupetit, Spectrum-preserving linear mappings between Banach algebras or JordanBanach algebras, J. London Math. Soc. 62 (2000), 917-924. 
[5] M. Brešar and P. Šemrl, Linear maps preserving the spectral radius, J. Funct. Anal. 142 (1996), 360-368.

[6] H. Halpern, Irreducible module homomorphisms of a von Neumann algebra into its center, Trans. Amer. Math. Soc. 140 (1969), 195-221.

[8] A. A. Jafarian and A. R. Sourour, Spectrum-preserving linear maps, J. Funct. Anal. 66 (1986), 255-261.

[7] P. Jordan, J. von Neumann and E. Wigner, On an algebraic generalization of the quantum mechanical formalism, Annals of Math. 35 (1934), 29-64.

[9] I. Kaplansky, Algebraic and Analytic Aspects of Operator Algebras, Amer. Math. Soc., Providence, RI, 1970.

[10] M. Mathieu, Spectrally bounded traces on $C^{*}$-algebras, Bull. Austral. Math. Soc. 68 (2003), 169-173.

[11] M. Mathieu, Spectrally bounded operators on simple $C^{*}$-algebras, Proc. Amer. Math. Soc. 132 (2004), 443-446.

[12] M. Mathieu and G. J. Schick, First results on spectrally bounded operators, Studia Math. 152 (2002), 187-199.

[13] M. Mathieu and G. J. Schick, Spectrally bounded operators from von Neumann algebras, J. Operator Theory 49 (2003), 285-293.

[14] M. Mathieu and A. R. Sourour, Hereditary properties of spectral isometries, Arch. Math. 82 (2004), 222-229.

[15] G. J. Murphy and T. T. West, Spectral radius formulae, Proc. Edinburgh Math. Soc. 22 (1979), 271-275.

[16] C. Pearcy and D. Topping, Sums of small numbers of idempotents, Mich. Math. J. 14 (1967), 453-465.

[17] G. K. Pedersen, Spectral formulas in quotient $C^{*}$-algebras, Math. Z. 148 (1976), 299-300.

[18] T. Ransford and M. White, Spectral characterization of algebraic elements, Bull. London Math. Soc. 33 (2001), 77-82.

[19] P. Šemrl, Spectrally bounded linear maps on B(H), Quart. J. Math. Oxford (2) 49 (1998), $87-92$.

[20] A. R. Sourour, The Gleason-Kahane-Żelazko theorem and its generalizations, in: Banach Center Publ. 30 (1994), 327-331.

[21] A. R. Sourour, Invertibility preserving linear maps on $L(X)$, Trans. Amer. Math. Soc. 348 (1996), 13-30.

[22] M. Takesaki, Theory of Operator Algebras I, Springer-Verlag, New York, 1979. 